\title{
APIGENIN CAUSES BIOCHEMICAL MODULATION, GLUT4 AND CD38 ALTERATIONS TO IMPROVE DIABETES AND TO PROTECT DAMAGES OF SOME VITAL ORGANS IN EXPERIMENTAL DIABETES
}

\author{
Chowdhury Mobaswar Hossain, Miltu Kumar Ghosh, \\ Bhabani Sankar Satapathy, Niladri Shekhar Dey and Biswajit Mukherjee \\ Department of Pharmaceutical Technology, Jadavpur University, Kolkata 700032, India
}

Received 2013-11-11; Revised 2013-11-26; Accepted 2014-01-13

\begin{abstract}
Diabetes mellitus gradually leads to dysfunction and failure of some vital organs specially the eyes, kidneys, pancreas, brain, heart, liver and lungs. The study was aimed to evaluate the antidiabetic potential of apigenin and its mechanistic role in controlling damages of vital tissues in streptozotocin-induced diabetic rats. Streptozotocin-induced diabetic rats were treated with apigenin and glipizide. Various biochemical changes, GLUT4 and CD38 protein expression patterns and histopathological alterations in some vital organs such as liver, kidneys and pancreas were investigated to compare the antidiabetic potentials of those two chemicals and to understand their capability to control the damages of the vital organs during diabetes. Effective control of blood glucose level along with the alteration of hepatic phase I and phase II drug metabolizing enzymes, antioxidant defense enzyme activities and lipid peroxidation level towards their normal values and enhanced GLUT4 translocation and downregulated CD38 expression by apigenin were observed. Apigenin was also found to prevent the deterioration of vital organs during diabetes. In conclusion, apigenin has predominant role in controlling blood glucose level along with the protection of vital organs eventually damaged during diabetes, by minimizing toxicities and associated diabetic complications in streptozotocin-induced diabetic rats and may explore as a potential antidiabetic agent in near future.
\end{abstract}

Keywords: Diabetes, Streptozotocin, Apigenin, GLUT4, CD38

\section{INTRODUCTION}

World Health Organisation has projected that the prevalence of Diabetes Mellitus (DM) will be 300 million by 2025 (Al-Amer et al., 2011). DM is a complex metabolic disorder involving alteration in carbohydrate, fat and protein metabolism (Patti and Corvera, 2010). There are two types of DM- type 1, Insulin Dependent Diabetes Mellitus (IDDM) and type 2, Noninsulin Dependent Diabetes Mellitus (NIDDM). IDDM is an autoimmune disease with complete destruction of pancreatic $\beta$-cells and requires insulin injection for its treatment, whereas the cause of NIDDM is insulin resistance and NIDDM is managed by the administration of synthetic drugs (Piero et al., 2012). NIDDM is characterized by persistent hyperglycemia with the changes of some biochemical parameters including lipid peroxidation, resulting from defects in insufficient insulin secretion, inefficient insulin function or both (Mukherjee et al., 2013). Streptozotocin (STZ) is used not only to induce type $1 \mathrm{DM}$ but also used to develop type 2 DM in animal model (King, 2012). It is reported that low dose STZ has been found to develop type 2 DM in rats (Wilson and Islam, 2012). An Corresponding Author: Biswajit Mukherjee, Department of Pharmaceutical Technology, Jadavpur University, Kolkata 700032, India Tel/Fax: +913324146677 
alteration in balance between the activity of endogenous pro-oxidative enzymes and antioxidant enzymes causes oxidative stress in cells (Giacco and Brownlee, 2010). Oxidative stress plays a pivotal role for the development of DM which increases complications with increased free radical formation (Karasu, 2010). Free radicals react with Polyunsaturated Fatty Acids (PUFA) of lipid membrane and cause lipid peroxidation (Barrera, 2012). Oxidative stress generates Reactive Oxygen Species (ROS), which produces toxic effect on cell development, growth and survival (Deavall et al., 2012). In hyperglycemia, oxidative stress is produced with the formation of Advanced Glycation End (AGE) product which has a strong association with the diabetic complications such as nephropathy, neuropathy, retinopathy and cardiovascular diseases (Villeneuve and Natarajan, 2010). GLUT4 is the insulin sensitive glucose transporter to skeletal muscle, adipose tissues and cardiac muscle. Reduction in insulin-mediated glucose uptake caused by decreasing GLUT4 protein translocation in Type 2 Diabetes (T2D) has been observed (Olson et al., 2012). Cluster of Differentiation 38 (CD38) enzyme degrades $\mathrm{NAD}^{+}$in tissue and promotes T2D and other metabolic syndrome (Escanade et al., 2013). Thus, facilitation of GLUT4 translocation by modulation of insulin signaling pathway and inhibition of $\mathrm{CD} 38$ protein can be potential therapeutic target for the treatment of T2D. Synthetic antidiabetic drugs for chronic use have been associated with various adverse effects, thus a renewed interest is growing for the developmental process of antidiabetic drug discovery and focus of research has been shifted to more efficacious compounds with less toxicity than the existing one. Apigenin, 4,5,7-trihydroxyflavone is a non-mutagenic flavone of a subclass of flavonoids with a very little toxicity (Cao et al., 2013). This compound has been reported to have anti-inflammatory, antioxidant and anti-carcinogenic properties (Liu et al., 2011). Apigenin has been reported to improve glucose tolerance through inhibition of microRNA maturation in miRNA103 transgenic mice (Ohno et al., 2013). Detailed investigation related to antidiabetic potential of apigenin still remains elusive. In the present study, effect of apigenin on STZ-induced diabetes in rats, histopathological alterations of different vital tissues such as liver, kidneys and pancreas, various biochemical alterations such as lipid peroxidation level, Phase I and phase II drug metabolizing enzymes, antioxidant defense enzymes and GLUT4 and CD38 protein expressions were investigated.

\section{MATERIALS AND METHODS}

\subsection{Chemicals And Reagents}

Apigenin from Cayman Chemicals (Michigan, USA) and STZ from Sigma Aldrich (Bangalore, India) were purchased. Catalase activity assay kit (Biovision, Milpitas, USA), Superoxide Dismutase (SOD) activity assay kit (Biovision, Mountain View, USA) and modified Lowry protein assay kit (Thermoscientific, Rockford, USA) were used. All other chemicals, reagents and solvents used in this study were of analytical grade.

\subsection{Development of Rat Diabetic Model}

Male Sprague-Dawley rats were purchased from Indian Institute of Chemical Biology, Kolkata, West Bengal. Initially the body weights of animals were approximately $110-130 \mathrm{~g}$. All animals were kept in polypropylene cages and housed in the university animal house at $25 \pm 1^{\circ} \mathrm{C}$ and $55 \%$ relative humidity environment with normal day and night photoperiod. Animals were fed standard rat diet and drinking water ad libitum. They have been acclimatized to the animal house environment for 2 weeks. The animal experiments were designed and conducted upon approval of the Animal Ethics Committee (AEC), Jadavpur University, Kolkata, India and strictly in accordance with the guidelines of AEC. STZ was freshly prepared in $0.1 \mathrm{M}$ citrate buffer $(\mathrm{pH}$ 4.5). T2D was induced experimentally by single intraperitoneal (i.p.) injection of low dose STZ (40 mg $\mathrm{kg}^{-1}$ ) to the rats, kept fasted overnight (Wang et al., 2013). Animals were unfed for $12 \mathrm{~h}$ before the estimation of fasting blood glucose. When the fasting plasma glucose level had become above $250 \mathrm{mg} \mathrm{dL}^{-1}$ in $72 \mathrm{~h}$ after STZ administration, STZ treated animals were considered as diabetic and all the experimental animals were then kept without any treatment for a period of 2 weeks. The apigenin treatment was started on 15 th day after STZ injection in the respective groups of rats.

\subsection{Experimental Design}

Apigenin was dissolved in water containing $0.1 \% \mathrm{v} / \mathrm{v}$ Dimethyl Sulfoxide (DMSO) for animal treatment according to the earlier report (Ohno et al., 2013). Absorption and elimination phase of apigenin via oral route is very slow and biological $t_{1 / 2}$ of apigenin is 91.8 h. As metabolism is slow, apigenin has general tendency to accumulate in tissue after oral administration. 
Apigenin was detected in rat plasma $30 \mathrm{~min}$ after its administration in rats via i.p., route (Gradolatto et al., 2005). Thus apigenin was administered here via i.p. route only. But glipizide, a sulfonylurea is absorbed efficiently after oral administration. Therefore, glipizide was administered by oral route only and the drug was taken as a commercially available standard antidiabetic drug for NIDDM. The animals were divided in six groups each of which contained six rats:

GroupI: Normal control group $(0.1 \% \mathrm{v} / \mathrm{v}$ DMSO as vehicle by i.p., route on alternate day for 28 days, i.e., on the same days on which group III rats were treated with apigenin)

GroupII:Diabetic control group (rats were made diabetic by a single i.p., administration of low dose STZ, $40 \mathrm{mg} \mathrm{kg}^{-1}$ body weight)

GroupIII:Diabetic group treated with apigenin $(1.5 \mathrm{mg}$ $\mathrm{kg}^{-1}$ body weight, i.p.,) (Tatsuta et al., 2000) on every alternate day for 28 days starting from day 15 after the STZ injection

GroupIV:Apigenin control group (only apigenin $1.5 \mathrm{mg}$ $\mathrm{kg}^{-1}$ body weight by i.p., route on every alternate day for 28 days, i.e., on the same days when the group III rats were treated with apigenin)

GroupV:Glipizide control group (only glipizide $5 \mathrm{mg}$ $\mathrm{kg}^{-1}$ body weight) orally daily for 28 days starting from day 15

GroupVI:Diabetic group treated with glipizide $(5 \mathrm{mg}$ $\mathrm{kg}^{-1}$ body weight) orally daily for 28 days starting from day 15 after the STZ injection

All the treatments were stopped on 42nd day. Daily food and water consumptions were observed and body weights were taken on weekly basis.

After $72 \mathrm{~h}$ of STZ administration, blood was drawn from tail vein of rats and fasting blood glucose was measured by glucometer (Contour blood glucose meter, Bayers Pharmaceuticals limited, Japan). Group II, group III and group VI rats were found diabetic. Apigenin (1.5 $\mathrm{mg} \mathrm{kg}{ }^{-1}$ body weight) was given by i.p. route to the rats belonged to group III and group IV for 28 days on every alternate day starting from day 15 after the STZ injection. Glipizide (5 $\mathrm{mg} \mathrm{kg}^{-1}$ body weight orally) was started to group V and group VI rats daily for 28 days. On the 43rd day, all rats were sacrificed by cervical dislocation. All animals were unfed for $12 \mathrm{~h}$ before being killed. Liver, kidneys, pancreas tissues and skeletal muscles (i.e., gastrocnemius muscles) were removed surgically and stored at $-80^{\circ} \mathrm{C}$ in a deep freezer (Ultra-Low Freezer, So-
Low, Cincinnati, Ohio, USA). Small pieces of different tissues as mentioned above were used for histopathological study. Remaining tissue samples were preserved at $-80^{\circ} \mathrm{C}$ in a deep freezer for estimation of some biochemical parameters such as lipid peroxidation, antioxidant defense enzymes, phase I and phase II drug metabolizing enzymes and for western blot analysis.

\subsection{Histopathological Studies}

For histopathological studies, some small pieces of liver, kidney and pancreas tissues of different experimental animals were fixed in $4 \%$ paraformaldehyde in phosphate buffer $(\mathrm{pH} 7.4)$ for $1 \mathrm{~h}$, subsequently processed and embedded in paraffin wax. Thin sections $(5 \mu)$ were cut and mounted on glass slides, processed for staining using haematoxylin-eosin $(\mathrm{H}$ and E) dye. The tissues were examined under Carl Zeiss light microscope (Axiostar plus, Jena, Germany).

\subsection{Determination of Average (Mean) Fasting Blood Glucose Level}

Blood samples were collected from tail vein of the different experimental rats on day 0 (on the day of STZ injection and before the administration of STZ injection), 7, 14, 21, 28, 35 and 42. Fasting blood sugar levels were estimated by glucometer according to the guidelines of the manufacturer and mean fasting blood glucose level of each group of rats was determined.

\subsection{Determination of Mean Body Weight}

Body weights of rats of different groups were taken on a weekly basis and mean body weight for each group of rats was determined.

\subsection{Biochemical Enzymes Assays}

\subsubsection{Preparation of Cytosolic And Microsomal Fraction}

Frozen liver tissue samples (stored at $-80^{\circ} \mathrm{C}$ ) of different experimental groups of rats were thawed, blotted quickly and weighed. The hepatic tissues were separately homogenized with ice-cold $0.1 \mathrm{~N} \mathrm{KCl}(\mathrm{pH}$ 7.4) in precooled teflon-coated glass homogenizer for few minutes to make $10 \% \mathrm{w} / \mathrm{v}$ tissue homogenate. The liver homogenate was centrifuged at $8000 \times \mathrm{g}$ for $15 \mathrm{~min}$ (at $4^{\circ} \mathrm{C}$ ) and then the supernatant was subjected to ultracentrifugation (WX-Ultra 90, T-890 rotor, Thermoscientific, Rockford, USA) at $78,000 \times \mathrm{g}$ for 90 min. The supernatant thus obtained, was used as cytosolic fraction and a portion of it was undergone ultracentrifugation at $1,05,000 \times \mathrm{g}$ for $1 \mathrm{~h}$ to obtain 
microsomes (precipitate) to assay UDP-glucuronosyl transferase (UDPGT), cytochrome P-450 (cyt P-450) content and to determine lipid peroxidation. The cytosolic fraction was used to determine glutathione Stransferase (GST) activity (Das et al., 2010).

\subsection{Assay For Cyt P-450, UDPGT And GST}

The total amount of microsomal cyt P-450 was estimated by the method as described (Zhang et al., 2012). The content of cyt P-450 was determined from the dithionite-reduced microsomes, with a molar extinction coefficient value of $91 / \mathrm{mM} / \mathrm{cm}$.

UDPGT activity towards p-nitrophenol was determined according to the available method (Andreassi et al., 2011; Zhang et al., 2013). The specific activity of UDPGT is expressed as activity/mg microsomal protein/min.

GST activity was measured (Olorunnisola et al., 2012) using 1-Chloro-2, 4-Dinitrobenzene (CDNB) as substrate. One unit of GST activity is defined as the amount of enzyme produces $1 \mathrm{mM}$ of CDNB-GSH conjugate/min under the conditions of the assay using molar extinction coefficient value of $9.6 / \mathrm{mM} / \mathrm{cm}$.

\subsection{Lipid Peroxidation}

Lipid peroxidation in liver was estimated by method available (Hossain et al., 2013). Malondialdehyde (MDA), a product of lipid peroxidation was determined spectrophotometrically by using Thiobarbituric AcidReactive Substances (TBARS). Total TBARS were expressed as MDA, using molar extinction coefficient for MDA of $1.56 \times 105 / \mathrm{cm} / \mathrm{M}$.

\subsection{Assay of Catalase and SOD}

The activity of catalase was estimated by catalase activity assay kit and SOD activity was measured by SOD activity assay kit.

Total protein was determined from cytosolic as well as microsomal fraction by modified Lowry protein assay kit.

\subsection{Isolation of Membrane}

Membrane samples were prepared from the liver cells and muscles according to available method (Moreno et al., 2012). Membrane samples were stored at $-70^{\circ} \mathrm{C}$ for analysis.

\subsection{Western Blot Analysis}

GLUT4 and CD38 expressions were assessed by Western blot. For GLUT4 content analysis, membrane samples were prepared from skeletal muscles and for
CD38 protein analysis, liver tissues were used. In Western blot analysis, $25 \mu \mathrm{g}$ of membrane protein per sample was subjected to Sodium Dodecyl Sulfate (SDS) polyacrylamide gel electrophoresis (Nagdas et al., 2013) in parallel with molecular weight markers (Rainbow Markers, Amersham, Buckinghamshire, England) on a $10 \%$ resolving gel. The separated proteins were then transferred to a nitrocellulose membrane in a semidry system (Trans-blot SD semidry transfer cell, Bio-Rad). For immunodetection, an electrochemiluminescenceWestern blotting kit was used, following the manufacturer's instructions (Amersham), using GLUT4 and CD38 rabbit polyclonal antibodies (Wak-Chemie Medical GmbH, Bad Soden, Germany) and a horseradish peroxidise-conjugated donkey anti-rabbit immunoglobulin secondary antibody (Amersham). Detection was done by the enhanced chemiluminiscence method and densitometric quantitiation was done by scanning of the autoradiographic GLUT4 and CD38 signals with the help of imageJ software (National Institute of Health, Baltimore, USA).

\subsection{Statistical Analysis}

Statistical calculations were performed with graphpad Instat version 3.0 (graphpad software, Inc., San Diego California, USA). Experimental data were statistically analyzed to find out the differences between the normal control rats and the other treated groups of rats and between the diabetic control group and the apigen in/glipizide treated diabetic groups of rats using Dunnett's t-test, following Analysis of Variance (ANOVA) to determine statistical level of significance with probability value $<0.05$.

\section{RESULTS}

\subsection{Average (Mean) Fasting Blood Glucose Level In Different Experimental Rats}

The mean blood glucose levels on $0,7,14,21,28$, 35 and 42nd day are shown in Fig. 1a. On day 14 after administration of STZ, all the rats belonging to group II (diabetic control rats), group III (diabetic rats treated with apigenin) and group VI (diabetic rats treated with glipizide) had significantly $(\mathrm{p}<0.05)$ higher blood glucose level than the blood glucose levels of them at day 0 . Till day 14 , rats were not treated with either apigenin or glipizide. The normal control rats did not show any significant variation in blood glucose level throughout the experimental period. 


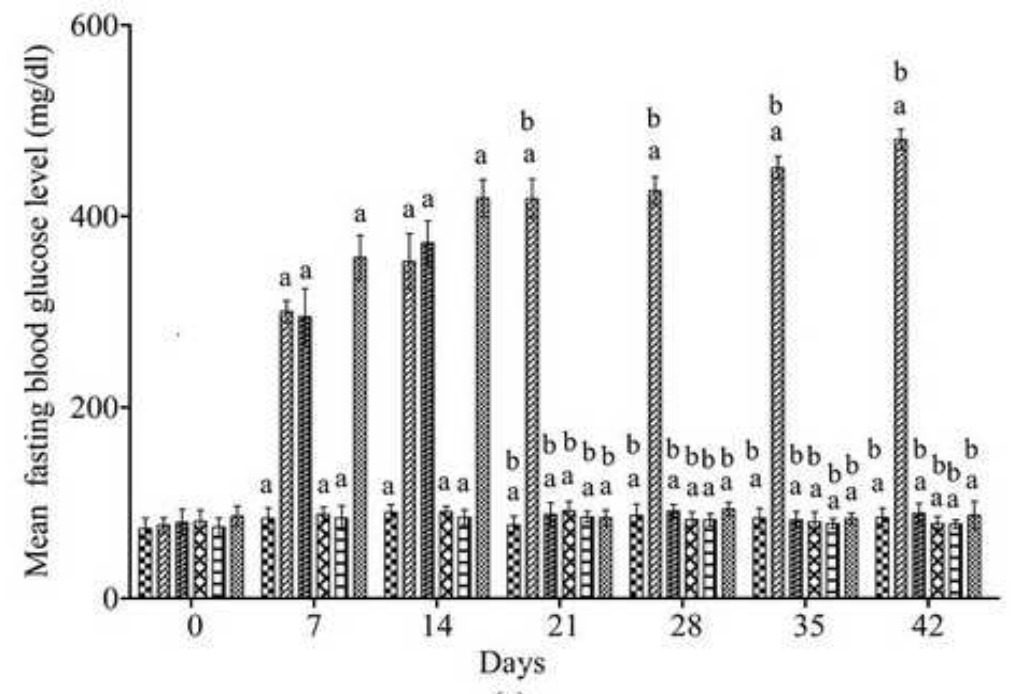

(a)



(b)

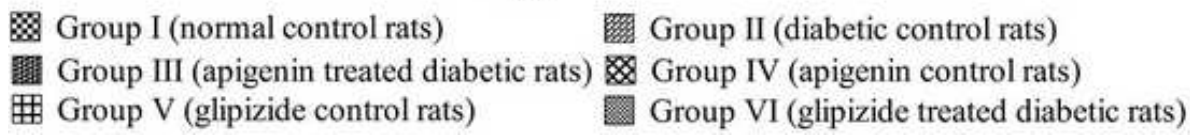

Fig. 1. (a) Mean fasting blood glucose levels for different experimental groups of rats. Each value represents mean $\pm S D(n=6$ ). ${ }^{a} \mathrm{p}<0.05,{ }^{b} \mathrm{p}<0.05$. ${ }^{\mathrm{a}}$ Statistical level of significance (using Dunnett's t-test) when fasting glucose levels on day 7 , day 14 , day 21, day 28, day 35 and day 42 were compared with the results at day 0. 'Statistical level of significance (using Dunnett's ttest) when fasting glucose levels on day 21 , day 28, day 35 and day 42 were compared with the results of day 14. (b) Mean body weight of different experimental groups of rats. Each value represents mean $\pm \operatorname{SD}(n=6) .{ }^{\mathrm{a}} \mathrm{p}<0.05,{ }^{\mathrm{b}} \mathrm{p}<0.05$. ${ }^{\mathrm{a}} \mathrm{Statistical}$ level of significance (using Dunnett's t-test) when mean body weights on day 7, day 14, day 21, day 28, day 35 and day 42 were compared with the data at day $0 .{ }^{\text {b }}$ Statistical level of significance (using Dunnett's t-test) when mean body weights on day 21 , day 28 , day 35 and day 42 were compared with those of day 14 
On the 42nd day, blood glucose levels were high in group II rats, but in group III and group VI rats, blood glucose levels were significantly $(\mathrm{p}<0.05)$ decreased $(7.33$-fold and 6.47fold, respectively) as compared to that in group II animals. Besides, fasting blood glucose levels in apigenin control rats (group IV) and glipizide control rats (group V) were lower than the blood glucose level in the normal rats.

\subsection{Average (Mean) Body Weight of Different Experimental Groups}

The average body weights of experimental rats on 0 , 7, 14, 21, 28, 35 and 42nd day are depicted in Fig. 1b. Before induction of diabetes, average body weights of all the rats were similar. Fourteen days after administration of STZ, the rats of group II, group III and group VI had significantly $(\mathrm{p}<0.05)$ lower mean body weights than those of the rats at the beginning of the experiment (i.e., day 0 ). On day 42 , average body weight was still low in the diabetic control rats (group II), but in group III and group VI rats, it increased significantly $(\mathrm{p}<0.05)$ as compared to group II rats. Group IV and group V rats had similar trend of changes in mean body weights as detected for group I (normal control) rats throughout the experimental period.

\subsection{Changes in Cyt P-450, Some Biochemical Enzyme Activities And Alteration in Lipid Peroxidation Level}

In group II rats, cyt P-450 content in liver was significantly $(\mathrm{p}<0.05)$ elevated (Fig. 2a). Upon treatment with apigenin and glipizide (group III and group VI rats respectively), cyt P-450 contents were significantly $(\mathrm{p}<0.05)$ decreased in those rats as compared to the diabetic control rats (group II). Cyt P-450 contents in the rats of group IV and group $\mathrm{V}$ were similar to that content in normal rats (group I).

Hepatic UDPGT activity was increased significantly $(\mathrm{p}<0.05)$ in diabetic control rats (group II) as compared to normal control rats (group I). UDPGT activity was found to be significantly low $(p<0.05)$ (Fig. 2b) in apigenin treated as well as glipizide treated diabetic control groups of rats (group III and group VI respectively) as compared to diabetic control rats. Group $\mathrm{IV}$ and group $\mathrm{V}$ rats did not show significant variation of UDPGT activity compared to normal control rats.

GST activity in liver was markedly enhanced in diabetic control rats (group II) compared to group I rats. But hepatic GST activity was significantly $(\mathrm{p}<0.05)$ reduced in rats of group III and group VI (Fig. 2c). No predominant alteration in the activity of GST was observed among group I, group IV and group V rats.
Unlike GST, a significant decrease of SOD activity was observed in diabetic control rats (group II) as compared to the normal control animals (Fig. 2d). Apigenin treated (group III) and glipizide treated rats (group VI) had significantly $(p<0.05)$ increased SOD activity than the diabetic control rats. SOD activity in group IV and group V rats was almost similar to that of group I rats.

Catalase activity in diabetic control rats was markedly decreased as compared to that of the normal control rats (Fig. 2e). Group III and group VI rats had significantly $\quad(\mathrm{p}<0.05)$ increased catalase activity compared to the diabetic control rats.

No predominant difference in catalase activity was observed among group I, group IV and group V rats.

A significant $(\mathrm{p}<0.05)$ elevation of MDA concentration was found in group II rats compared to that of the normal control rats. Lipid peroxidation (as meausured by MDA content) was increased following STZ administration. However, there were significant reductions in lipid peroxidation level upon apigenin and glipizide treatments in group III and group VI rats, respectively (Fig. 2f). No alteration in lipid peroxidation was observed in group IV and group V rats.

\subsection{Histopathological Observations in Liver, Kidneys And Pancreas}

The STZ-induced diabetic control rats had hepatocytes with many pycnotic nuclei with dilatation of sinusoidal spaces (Fig. 3a, b). In apigenin treated diabetic rats, nuclei and sinusoidal spaces were more or less similar to those of normal liver (Fig. 3c). Liver of diabetic rats after the treatment with glipizide has shown hepatocytes similar to those of normal control rats (Fig. 3d). As compared to the structure of Bowman's capsules and renal tubules of normal control rats, the diabetic rats had swelled (expanded) Bowman's capsules along with occasional damage of glomerulus and nephritic cells as observed in histological kidney sections (Fig. 4a, b). Apigenin treatment in diabetic rats has shown to reverse the swelled (expanded) Bowman's capsules in kidneys towards the normal one (Fig. 4c). Kidneys of diabetic rats after treatment with glipizide have shown Bowman's capsules almost similar to those of normal rats (Fig. 4d). Normal control rats have shown normal islets with the patches of abundant $\beta$-cells. In diabetic group, islets of $\beta$-cells were severely damaged. Again, atrophy of $\beta$-cells and degenerative changes in the islets were observed in the pancreas of diabetic rats (Fig. 5a, b). 

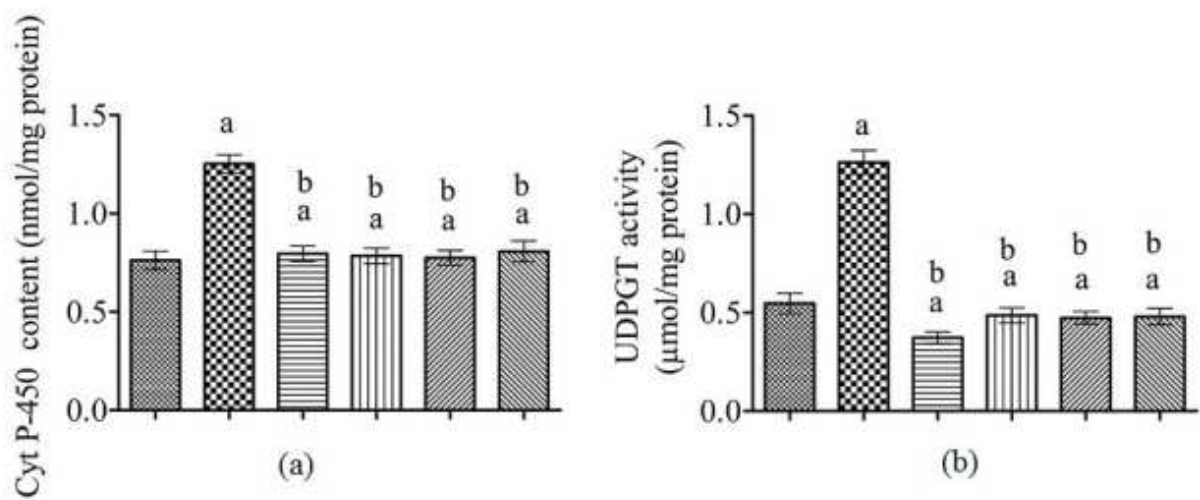

(b)

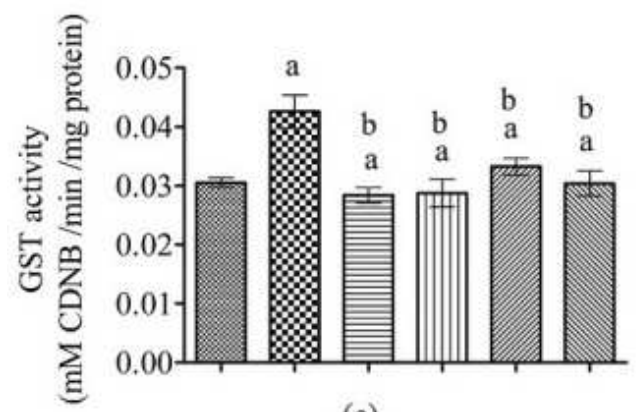

(c)



(d)

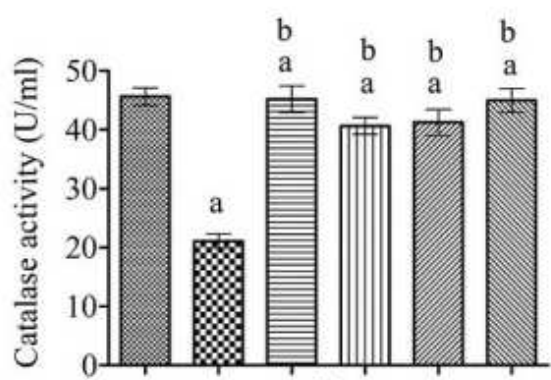

(e)

Group I (normal control rats)

Group III (apigenin treated diabetic rats)

Group V (glipizide control rats)

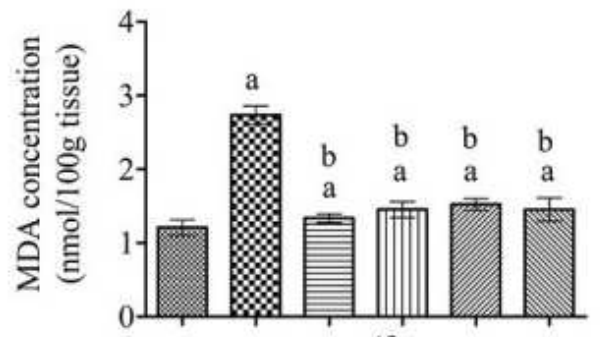

(f)

Q Group II (diabetic control rats)

Group IV (apigenin control rats)

Group VI (glipizide treated diabetic rats)

Fig. 2. Assay of biochemical parameters in different experimental groups of rats. (a) Cyt-450 content. (b) UDPGT activity (c) GST activity (d) SOD activity (e) Catalase activity (f) Lipid peroxidation level. Each value represents mean \pm SD $(n=6)$. ${ }^{a} p<0.05$, ${ }^{b} \mathrm{p}<0.05$. 'Statistical level of significance (using Dunnett's t-test) of the data obtained from group II, III, IV, V and VI rats when compared with those of group I rats. 'Statistical level of significance (using Dunnett's $t$-test) of the data obtained from group III, IV, V and VI rats when compared with the data of group II rats

Interestingly, apigenin treatment preserved compact islets in pancreas and pancreatic $\beta$-cells which were found almost similar to those of normal control rats (Fig. 5c). Glipizide treated diabetic rats have shown pancreatic $\beta$ - cells nearly normal (Fig. 5d). In apigenin control and glipizide control rats histology of liver, kidneys and pancreas tissues did not show any predominant differences as compared to normal control rats (data not shown). 


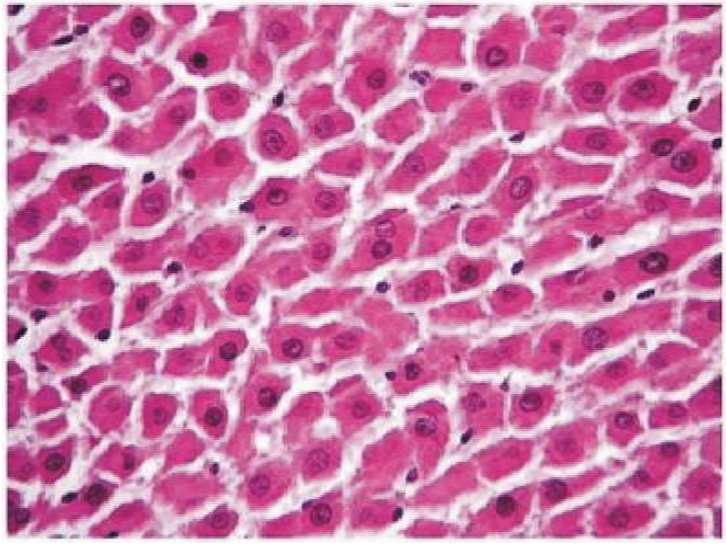

(a)

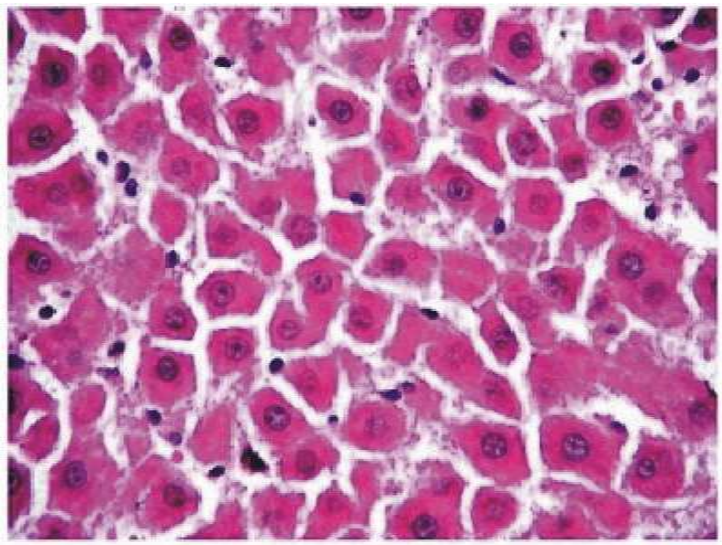

(c)

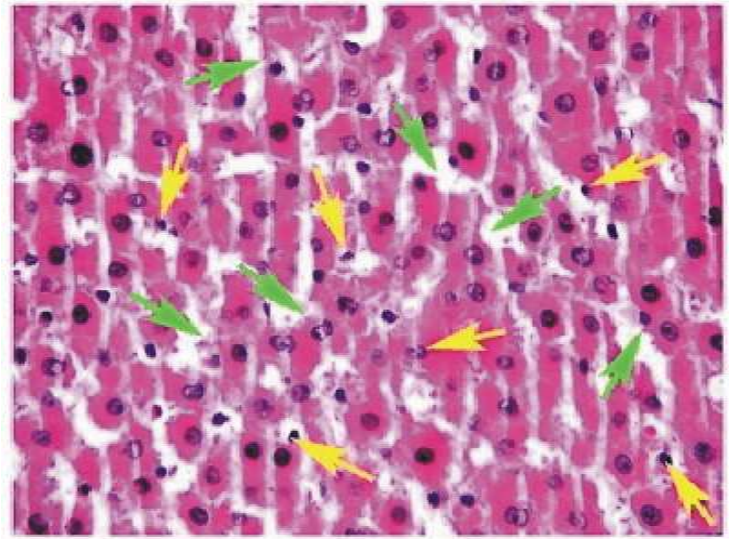

(b)

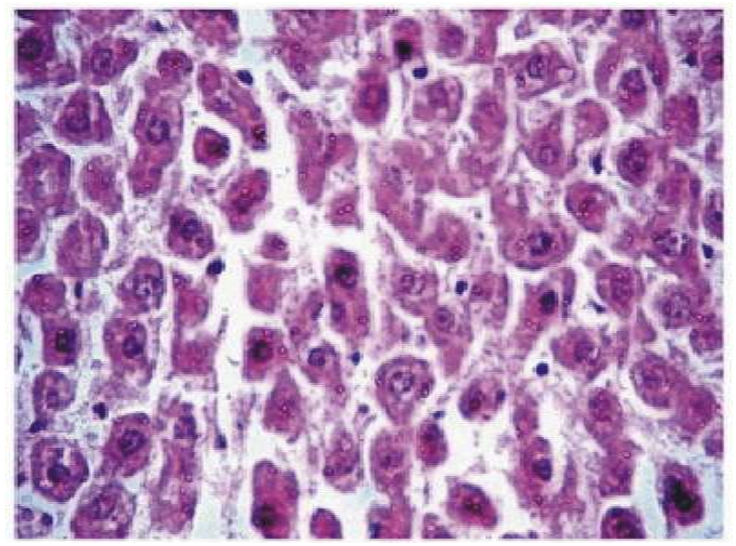

(d)

Fig. 3. Histopathological observations of different experimental rat livers (400×magnification), (a) Liver section of normal control rats showing normal hepatocytes, (b) Liver section of diabetic control rats showing many hepatocytes containing pycnotic nuclei (shown by yellow arrow) and dialated sinusoidal space (shown by green arrow) in the hepatic tissue, (c) Liver section of diabetic rats treated with apigenin showing normal hepatocytes, (d) Liver section of diabetic group treated with glipizide showing normal hepatocytes



(a)



(b) 




(c)



(d)

Fig. 4. Histopathological observations of different experimental rat kidney sections (400xmagnification), (a) Kidney section of normal control rats showing normal Bowman's capsule and nephritic cells, (b) Kidney section of diabetic control rats showing swelled (expanded) Bowman's capsule with occasional damages of glomerulus (shown by green arrow) and nephritic cells (shown by yellow arrow), (c) Kidney section of diabetic rats treated with apigenin showing Bowman's capsule and nephritic cells towards the normal one, (d) Kidney section of diabetic rats treated with glipizide showing Bowman's capsule and nephritic cells similar to that of normal rats

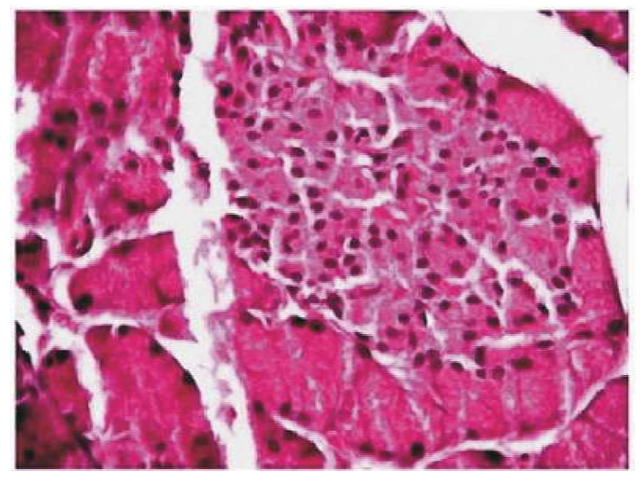

(a)

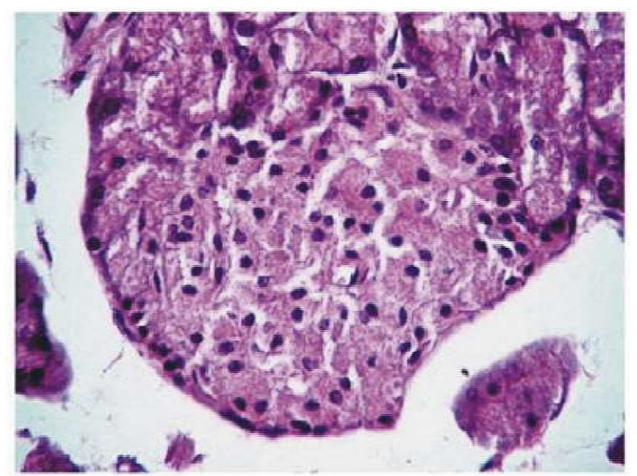

(c)



(b)



(d)

Fig. 5. Histopathological observations of experimental rat pancreas, (a) Pancreatic section of normal control rat showing normal islets and abundant pancreatic $\beta$-cells (1000×magnification), (b) Pancreatic section of diabetic control rat showing atrophy $\beta$ cells and degenerative changes in islets, shown by green arrows (400×magnification), (c) Pancreatic section of a diabetic rat treated with apigenin showing preservation of compact islets and $\beta$-cells (1000×magnification), (d) Pancreatic section of a diabetic rat treated with glipizide showing recovery of islets and $\beta$-cells to almost normal (400xmagnification) 


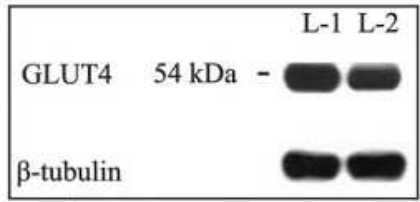

(a)

L-1: Diabetes + Apigenin

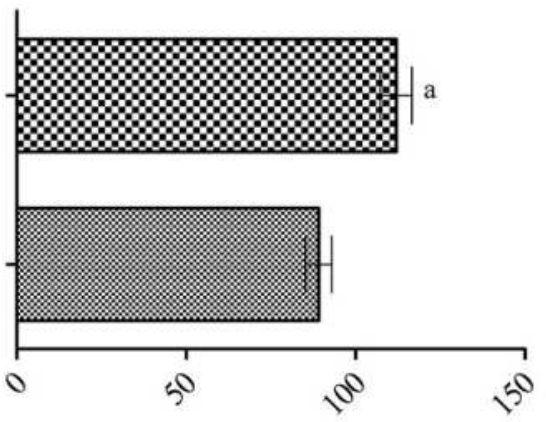

Band intensity of GLUT4 (\%)

(c)

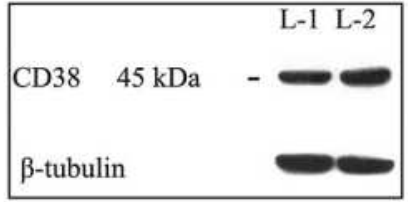

(b)

L-2: Diabetic control

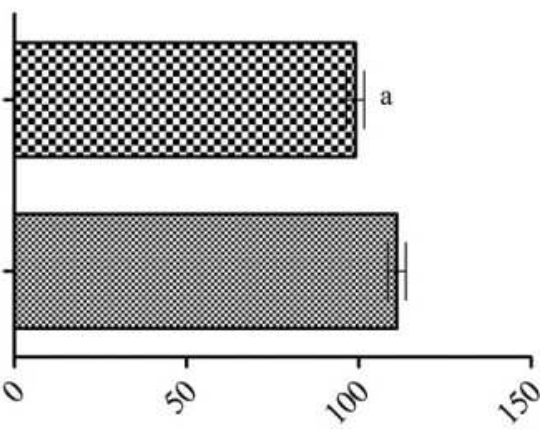

Band intensity of CD38 (\%)

(d)

$\mathbf{8}$ Diabetic rats treated with apigenin

\section{Diabetic control rats}

Fig. 6. Western blot analysis of GLUT4 and CD38 protein expression in apigenin treated diabetic rats and diabetic control rats. Total protein $(25 \mu \mathrm{g})$ from each cell lysate was analysed by Western blotting by anti-GLUT4 and anti-CD antibodies. $\beta$-tubulin was used to ensure equivalent loading of the gel incubating the same membrane with $\beta$-tubulin antibody after stripping, (a) Band of GLUT4 expression in the skeletal muscles of apigenin treated diabetic rats and diabetic control rats, (b) Band of CD38 expression in the liver of apigenin treated diabetic rats and diabetic control rats, (c) Densitometric scanning (percentage of band intensity) of GLUT4 bands in apigenin treated diabetic rats and diabetic control rats using imageJ software, (d) Densitometric scanning (percentage of band intensity) of CD38 bands in apigenin treated diabetic rats and diabetic control rats using imageJ software. Data are expressed as percentage increase or decrease of intensity of band. Each value represents mean $\pm \mathrm{SD}(\mathrm{n}=6) .{ }^{\mathrm{a}} \mathrm{p}<0.05$. ${ }^{\mathrm{a}}$ Statistical level of significance (using Dunnett's $t$-test) when data of diabetic control rats are compared with the data of apigenin treated diabetic rats

\subsection{GLUT4 And CD38 Expression in Apigenin Treated Diabetic Rats And Diabetic Control Rats}

Apigenin treated diabetic rats showed approximately $27 \%$ significant $(\mathrm{p}<0.05)$ increase of GLUT4 translocation in skeletal muscles than that of diabetic control rats (Fig. 6a and c). A significant $(\mathrm{p}<0.05)$ decrease (approximately 11\%) of CD38 expression was observed in apigenin treated diabetic rats than that of diabetic rats (Fig. 6b and d).

\section{DISCUSSION}

Oxidative stress in DM results in macro-and microvascular complications exerting toxicities on different vital organs (Andreassi et al., 2011). STZ causes destruction of $\beta$-cells of the islets and reduction in insulin release (Ning et al., 2011). The structural and biochemical functions of liver and kidneys may also be affected by alteration of insulin concentration which inhibits rapid glucose uptake and storage as glycogen in liver (Seyer et al., 2013). It is well recognized that flavonoids are able to improve diabetic subject by decreasing blood glucose levels (Rauter et al., 2010). In our study, a significant decrease of fasting blood glucose level upon apigenin administration in STZ-induced diabetic rats indicates the potential of the flavonoid to ameliorate DM. Glipizide, a popular antidiabetic drug, was used to compare the antihyperglycemic efficacy of apigenin in diabetic rats. STZ treatment usually caused a significant reduction of body weight and our findings 
have supported the earlier published report in this case (Yin et al., 2011). Decreased body weight during diabetes generally occurs due to excessive breakdown of tissue proteins (Bonaldo and Sandri, 2013). Apigenin significantly improved the decreased body weight in diabetic rats. Apigenin and glipizide effectively decreased elevated blood glucose in diabetic rats.

Cyt P-450, an isoenzyme belonging to a supergene family of heme-containing oxidase, involved in the phase I drug metabolism and plays an important role in detoxification of many xenobiotics and endogeneous compounds in hepatocytes (Hossain et al., 2013). Content of cyt P-450 has been reported to increase in the liver microsomes of diabetic subject (Hossain et al., 2013). In our study, apigenin treatment in diabetic control rats has shown to reduce enhanced content of cyt $\mathrm{P}-450$ as compared to the diabetic control rats, indicating the involvement of cyt P-450 enzymes during the development and progression of diabetes and apigenin might take up the role to reduce enhanced isoenzyme level in diabetic rats towards the normal value.

Antioxidant defense against ROS are reduced in T2D (Lemos et al., 2012). A balance between the formation of free radicals and the endogenous defense system is destroyed, which is considered to be the cause of various diseases including diabetes, cancer and cardiovascular complications (Villeneuve and Natarajan, 2010). The present study has shown a significant reduction in lipid peroxidation with apigenin treatment in diabetic rats as compared to diabetic control rats. The result suggests that like many other flavonoids, apigenin also provides antioxidant effect. UDPGT and GST, two important phase II enzymes, play important roles in metabolism of xenobiotics and protect liver against xenobioticsinduced hepatotoxicity (Wang et al., 2012). A report suggests that UDPGT activity was increased in liver of diabetic mice due to physicochemical changes in lipid compositions and fluidity of microsomal membranes (Braun et al., 1998). The present study has shown that the hepatic UDPGT activity towards p-nitrophenol were decreased in group III and group VI rats, whereas an elevated level of the enzyme was observed in group II rats (diabetic control). A similar trend of effect was observed in GST activity. An increase in GST activity indicates the apparent stress in hepatocytes in terms of increased concentrations of cellular metabolites that might accumulate under the diabetic conditions (Ballatori et al., 2009). Again, elevated level of UDPGT and GST in group II rats have been brought back to the normal or near normal values after the apigenin administration. Thus the data support for apigenin mediated clearence of enhanced accumulation of cellular metabolites that produce stress in diabetes.

STZ produces ROS $\left(\mathrm{O}_{2}^{-}\right.$and $\left.\mathrm{H}_{2} \mathrm{O}_{2}\right)$ by the xanthine oxidase system of pancreatic cells and causes methylation and fragmentation of DNA in rat liver, kidneys and pancreatic islets (El-Awdan et al., 2013). SOD catalyses the conversion of $\mathrm{O}_{2}^{-}$to $\mathrm{H}_{2} \mathrm{O}_{2}$ whereas catalase catalyses the conversion of $\mathrm{H}_{2} \mathrm{O}_{2}$ to $\mathrm{H}_{2} \mathrm{O}$ (Sharma et al., 2012). In the present study, SOD and catalase activities were found to decrease in diabetic control rats (group II), whereas in apigenin and glipizide treated diabetic rats (group III and group VI respectively), the activities of those two enzymes were found to enhance towards normal values. Elevated MDA concentration may have link with enhanced ROS generation, which modulates activities of the potential antioxidant enzymes. In the present study, apigenin might have scavenged free radicals and/or activated antioxidant defense enzymes. Free radical scavenging property of apigenin depends on the number of $\mathrm{OH}$ groups present at 4, 5 and 7th positions of its C-ring structure (Sharma et al., 2012). The antioxidant property of apigenin has been reported due to double bond between the $\mathrm{C}_{2}$ and $\mathrm{C}_{3}$ carbon atoms of the C-ring and presence of oxo group in 4th position of C-ring (Jeyabal et al., 2005) (Fig. 7). Our data also suggests that apigenin has strong antioxidant potential.

The pathological alterations in the liver of diabetic rats have been reported earlier (Liu et al., 2013). In our study, hepatocytes of diabetic control rats have shown scattered pycnotic nuclei and dilatation of sinusoidal spaces. Whereas apigenin treated diabetic rats (group III) significantly improved the degenerative changes of hepatocytes to almost normal. Apigenin has shown better preservation of hepatocytes when compared to diabetic rats treated with glipizide. In diabetes, degradation of liver glycogen and gluconeogenesis are increased while glucose utilization is inhibited (Lisman et al., 2010). The pathological alteration of diabetic liver is due to glycosylation of proteins leading to abnormalities in hepatic ultrastructures (Ntimbane et al., 2009).

Diabetic nephropathy is the progressive and fatal impact of DM which is primarily manifested by microalbuminuria causing albuminuria and finally renal failure (Heras et al., 2012). Our findings reveal that in the kidneys of diabetic control rats, Bowman's capsules and glomeruli were swelled and damage of nephritic cells was prominent. The excellent recovery of kidney cell architecture was found in apigenin treated diabetic rats. 


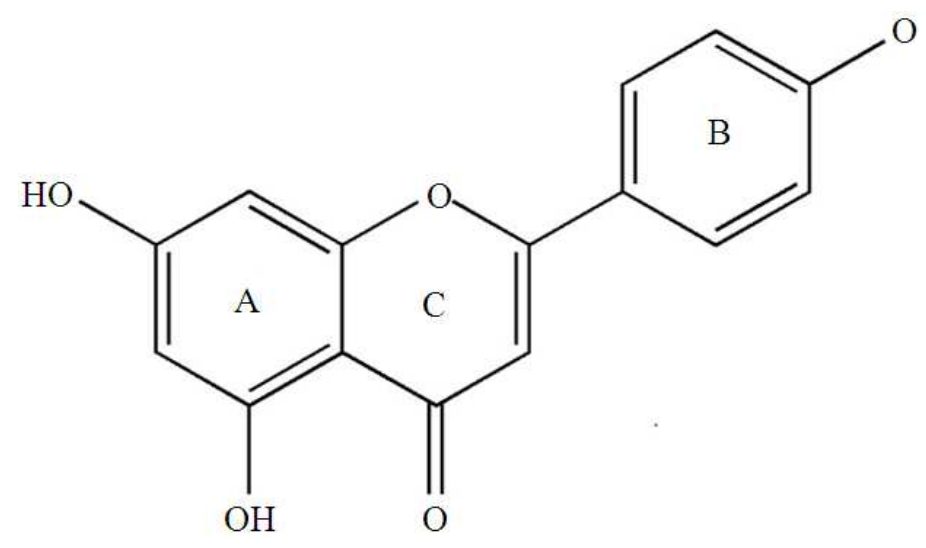

Fig. 7. Chemical structure of apigenin (4', 5, 7- trihydroxyflavone)

Previous studies have reported that STZ induces NO liberation, causes alkylation and fragmentation of DNA, leading to apoptosis (Espino et al., 2011). In the present study, pancreatic $\beta$-cells in diabetic control rats were severely damaged and destroyed. Upon apigenin treatment the normal structure of pancreatic $\beta$-cells was significantly regained and preserved. Glipizide treated diabetic rats (group VI) also partially maintained or reverted back the $\beta$-cells structure, but action of apigenin was more prominent.

GLUT4 is insulin-sensitive glucose transporter, mainly expressed in skeletal muscles, adipocytes and cardiac muscles. Defects in GLUT4 trafficking are likely to contribute to the insulin resistance in skeletal muscle from patients with T2D (Choi and Kim, 2010). We have shown in our study that apigenin facilitates GLUT4 translocation in skeletal muscles of apigenin treated diabetic rats than that of the diabetic control rats. Our study highlights that possible mechanism of apigeninmediated stimulation of GLUT4 translocation occurs in muscles of type 2 diabetic rats, either by activation of insulin signaling pathway or AMP-activated protein kinase pathway (Prasad et al., 2010).

CD38 enzyme is a $\mathrm{NAD}^{+}$ase which degrades $\mathrm{NAD}^{+}$ in tissue and promotes metabolic syndrome (Escanade et al., 2013). Our findings provide mechanistic evidence that apigenin downregulates CD38 expression in type II diabetic rats. This data is consistent with the apigenin-mediated improvement of glucose homeostasis via SIRT1 activation and promotion of fatty acid oxidation along with reduction of lipids accumulation (Escanade et al., 2013). Importantly, our study reveals that pharmacological downregulation or inhibition of CD38 expression at the molecular level can be a therapeutic approach to treat T2D.

\section{CONCLUSION}

Apigenin appears to have a strong antidiabetic potential. It modulates lipid peroxidation level, phase I (cyt P-450) and phase II (UDPGT and GST) drug metabolizing enzymes and antioxidant defense enzymes (SOD and catalase) in favour of improving T2D. Apigenin preserves the cellular architecture of vital tissues (such as liver, kidneys and pancreas) towards normal in STZ-induced diabetic rats. It did not produce any damage of the vital tissues as studied for normal rats. Furthermore, enhanced GLUT4 translocation upon apigenin treatment suggests more glucose lowering as well as $\beta$-cell preserving efficacy. Downregulation of CD38 with apigenin revealed that apigenin may be effective in improving glucose homeostasis and metabolic diseases such as T2D, obesity and obesity related diseases. So apigenin may be projected as a potential antidiabetic agent in near future and it may protect vital organs which are normally damaged during diabetes since persistent hyperglycemia inevitably damages vital organs. Apigenin controls hyperglycemia and both the compounds, apigenin and glipizide not only control blood glucose level towards normal, but also ameliorate the deterioration of vital tissues due to hyperglycaemic condition.

\section{ACKNOWLEDGEMENT}

Researchers are indebted to the grant of Department of Science and Technology (Government of India), Grant no. DST/Inspire Fellowship/2010/ 87. 


\section{REFERENCES}

Al-Amer, R.M., M.M. Sobeh, A.A. Zayed and H.A. AlDomi, 2011. Depression among adults with diabetes in Jordan: Risk factors and relationship to blood sugar control. J. Diab. Complicat., 25: 247-252. PMID: 21601482

Andreassi, M.G., R. Barale, P. Iozzoand and E. Picano, 2011. The association of micronucleus frequency with obesity, diabetes and cardiovascular disease. Mutagenesis, 26: 77-83. PMID: 21164186

Ballatori, N., S.M. Krance, S. Notenboom, S. Shi and K. Tieu et al., 2009. Glutathione dysregulation and the etiology and progression of human diseases. Biol. Chem., 390: 191-214. PMID: 19166318

Barrera, G., 2012. Oxidative stress and lipid peroxidation products in cancer progression and therapy. Oncology, 2012: 1-21. PMID: 23119185

Bonaldo, P. and M. Sandri, 2013. Cellular and molecular mechanisms of muscle atrophy. Dis. Model Mech., 6: 25-39. DOI: 10.1242/dmm.010389

Braun, L., M.J. Coffey, F. Puskás, T. Kardon and G. Nagy et al., 1998. Molecular basis of bilirubin UDPglucuronosyltransferase induction in spontaneously rats, acetone-treated rats and starved rats. Biochem. J., 336: 587-592. PMID: 9841869

Cao, X., B. Liu, W. Cao, W. Zhang and F. Zhang et al., 2013. Autophagy inhibition enhances apigenininduced apoptosis in human breast cancer cells. Chin. J. Cancer. Res., 25: 212-222. PMID: 23592903

Choi, K. and Y. B. Kim, 2010. Molecular mechanism of insulin resistance in obesity and type 2 diabetes. Korean J. Int. Med., 25: 119-129. PMCID: PMC2880683

Das, T., F. Patra and B. Mukherjee, 2010. Effect of antisense oligomer in controlling c-raf.1 overexpression during diethylnitrosamine-induced hepatocarcinogenesis in rat. Cancer Chemother. Pharmacol., 65: 309-318. PMID: 19499222

Deavall, D.G., A.M. Elizabeth, M.H. Judith and R. Ruth, 2012. Drug-induced oxidative stress and toxicity. J. Toxicol., 2012: 1-13. DOI: 10.1155/2012/645460

El-Awdan, S.A., J.A.A. Jaleel and D.O. Saleh, 2013. Grape seed extract attenuates hyperglycaemiainduced in rats by streptozotocin. Pharmacy Cairo Univ. DOI: 10.1016/j.bfopcu.2013.05.003

Escanade, C., V. Nin, N.L. Price, V. Capellini and A.P. Gomes et al., 2013. Flavonoid apigenin is an inhibitor of the $\mathrm{NAD}^{+}$ase CD38 implications for cellular $\mathrm{NAD}^{+}$metabolism, protein acylation and treatment of metabolic syndrome. Diabetes, 62: 1084-1093. PMID: 23172919
Espino, J., J. A. Pariente and A. B. Rodriguez, 2011. Role of melatonin on diabetes-metabolic disorders. World. J. Diabetes, 2: 82-91. PMCID: PMC3158876

Giacco, F. and M. Brownlee, 2010. Oxidative stress and diabetic complications. Circ. Res., 107: 1058-1070. PMID: 21030723

Gradolatto, A., J. P. Basly, R. Berges, C. Teyssier and M.C. Chagnon et al., 2005. Pharmacokinetics and metabolism of apigenin in female and male rats after a single oral administration. Drug Metab. Dispos., 33: 49-54. PMID: 15466493

Heras, M.M., N.D.C. Rodriguez and J.F.N. Gonzalez, 2012. The renin-angiotensin-aldosterone system in renal and cardiovascular disease and the effects of its pharmacological blockade. J. Diabetes Metab., 3: 1-24. DOI: 10.4172/2155-6156.1000171

Hossain, C.M., B.S. Satapathy, L. Mondal and S. Chakraborty, 2013. Effect of streptozotocin-induced hyperglycemia on the progression of hepatocarcinogenesis in rats. Am. J. Pharmacol. Toxicol., 8 : 170-178. DOI: 10.3844/ajptsp.2013.170.178

Jeyabal, P.V.S., M.B. Syed, M. Venkataraman, J.K. Sambandham, D. Sakthisekaran, 2005. Apigenin inhibits oxidative stress-induced macromolecular damage in N-Nitrosodiethylamine (NDEA)-induced hepatocellular carcinogenesis in wistar albino rats. Mol. Carcinog., 44: 11-20. PMID: 15924350

Karasu, C., 2010. Glycoxidative stress and cardiovascular complications in experimentally-induced diabetes: Effects of antioxidant treatment. Open Cardiovasc. Med. J., 4: 240-256. PMID: 21270942

King, A.J.F., 2012. The use of animal models in diabetes research. Br. J. Pharmacol., 166: 877-894. PMID: 22352879

Lemos, E.T.D., J. P. Pinheiro and F. Reis, 2012. Regular physical exercise as a strategy to improve antioxidant and anti-inflammatory status: Benefits in type 2 diabetes mellitus. Oxid. Med. Cell. Longev., 2012: 1-15. DOI: 10.1155/2012/741545

Lisman, T., S.H. Caldwell, A.K. Burroughs, P.G. Northup and M. Senzolo, 2010. Hemostasis and thrombosis in patients with liver disease: The ups and downs. J. Hepatol., 53: 362-371. PMID: 20546962

Liu, F., M. Xie, D. Chen, J. Li and W. Ding, 2013. Effect of $\mathrm{V}^{\mathrm{IV}} \mathrm{O}$ (dipic-Cl) $\left(\mathrm{H}_{2} \mathrm{O}\right)_{2}$ on lipid metabolism disorders in the liver of STZ-induced diabetic rats. J. Diabetes Res., 2013: 1-10. DOI: $10.1155 / 2013 / 956737$ 
Liu, Q., X. Chen, G. Yang, X. Min and M. Deng, 2011. Apigenin inhibits cell migration through MAPK pathways in human bladder smooth muscle cells. Biocell, 35: 71-79. PMID: 22423483

Moreno, P., B. Nuche-Berenguer, I. Gutierrez-Rojas, A. Acitores and V. Sancho et al., 2012. Normalizing action of exendin-4 and GLP-1 in the glucose metabolism of extrapancreatic tissues in insulinresistant and type 2 diabetic states. J. Mol. Endocrinol., 48: 37-47. DOI: 10.1530/JME-11-0127

Mukherjee, B., C.M. Hossain, L. Mondal, P. Paul and M.K. Ghosh, 2013. Obesity and insulin resistance: An abridged correlation. Lipid Insights, 6: 1-11. DOI: 10.4137/LPI.S10805

Nagdas, S.K., T. Buchanan, S. McCaskill, J. Mackey and G.E. Alvarez et al., 2013. Isolation of a calciumbinding protein of the acrosomal membrane of bovine spermatozoa. Int. J. Biochem. Cell Biol., 45: 876-884. PMID: 23376657

Ning, Y., W. Zhen, Z. Fu, J. Jiang and D. Liu et al., 2011. Ranolazine increases $\beta$-cell survival and improves glucose homeostasis in low-dose streptozotocininduced diabetes in mice. J. Pharmacol. Exp. Ther., 337: 50-58. PMID: 21228065

Ntimbane, T., B. Comte, G. Mailhot, Y. Berthiaume and V. Poitout et al., 2009. Cystic fibrosis-related diabetes: From CFTR dysfunction to oxidative stress. Clin. Biochem. Rev., 30: 153-177. PMID: 20011209

Ohno, M., C. Shibata, T. Kishikawa, T. Yoshikawa and A. Takata et al., 2013. The flavonoid apigenin improves glucose tolerance $t$ hrough inhibition of microRNA maturation in miRN A103 transgenic mice. Sci. Rep., 3: 2553-2559. DOI: $10.1038 /$ srep02553

Olorunnisola, O.S., G. Bradley and A.J. Afolayan, 2012. Protective effect of $T$. violacea rhizome extract against hypercholesterolemia-induced oxidative stress in wistar rats. Molecules, 17: 6033-6045. DOI: $10.3390 /$ molecules 17056033

Olson, A.L., 2012. Regulation of GLUT4 and insulindependent glucose flux. Mol. Biol., 2012: 1-12. DOI: $10.5402 / 2012 / 856987$

Patti, M.E. and S. Corvera, 2010. The role of mitochondria in the pathogenesis of type 2 diabetes. Endocr. Rev., 31: 364-395. PMID: 20156986

Piero, N.M., N.J. Murugi, O.R. Okoth, M.A. Jalemba and N.N.J. Joseph et al., 2012. Prevention of type I diabetes mellitus: The role of immune interventions. J. Clin. Cell. Immunol., S2: 1-6. DOI: 10.4172/2155-9899

Prasad, C.N.V., T. Anjana, A. Banerji and G. Anilkumar, 2010. Gallic acid induces GLUT4 translocation and glucose uptake activity in 3T3-L1 cells. FEBS Lett., 584: 531-536. PMID: 19962377
Rauter, A. P., A. Martins, C. Borges, H.M. Filipe and R. Pinto et al., 2010. Antihyperglycemic and protective effects of flavonoids on streptozotocin-induced diabetic rats. Phytother. Res., 24: S133-S138. PMID: 20309949

Seyer, P., D. Vallois, C. Poitry-Yamate, F. Schutz and S. Metref et al., 2013. Hepatic glucose sensing is required to preserve $\beta$-cell glucose competence. $\mathrm{J}$. Clin. Invest., 123: 1662-1676. PMID: 23549084

Sharma, P., A.B. Jha, R.S. Dubey and M. Pessarakli, 2012. Reactive oxygen species, oxidative damage and antioxidative defense mechanism in plants under stressful conditions. J. Bot., 2012: 1-26. DOI: $10.1155 / 2012 / 217037$

Tatsuta, M., H. Iishi, M. Baba, K. Murata and M. Mukai et al., 2000. Suppression by apigenin of peritoneal metastasis of intestinal adenocarcinomas induced by azoxymethane in Wistar rats. Clin. Exp. Metastasis, 18: 657-662. PMID: 11827069

Villeneuve, L.M. and R. Natarajan, 2010. The role of epigenetics in the pathology of diabetic complications. Am. J. Physiol. Renal. Physiol., 299: F14-F25. PMID: 20462972

Wang, L., G. Duan, Y. Lu, S. Pang and X. Huang et al., 2013. The effect of simvastatin on glucose homeostasis in streptozotocin induced type 2 diabetic rats. J. Diabetes Res., 2013: 1-5. PMID: 23671864

Wang, Y., X. Peng, W. Xu, Y. Luo and W. Zhao et al., 2012. Transcript and protein profiling analysis of OTA-induced cell death reveals the regulation of the toxicity response process in Arabidopsis Thaliana. J. Exp. Bot., 63: 2171-2187. PMID: 22207617

Wilson, R.D. and M.S. Islam, 2012. Fructose-fed streptozotocin-injected rat: An alternative model for type 2 diabetes. Pharmacol. Rep., 64: 129-139. PMID: 22580529

Yin, P., S. Zhao, S. Chen, J. Liu and L. Shi et al., 2011. Hypoglycemic and hypolipidemic effects of polyphenols from burs of Castanea mollissima blume. Molecules, 16: 9764-9774. PMID: 22116046

Zhang, C.L., T. Zeng, X.L. Zhao and K.Q. Xie, 2013. Garlic oil attenuated nitrosodiethylamine-induced hepatocarcinogenesis by modulating the metabolic activation and detoxification enzymes. Int. J. Biol. Sci., 9: 237-245. DOI: 10.7150/ijbs.5549

Zhang, Y., Y. Li and Q. Li, 2012. Inhibition of cytochrome $\mathrm{P} 4503 \mathrm{~A}$ in rat liver by the diorganotin (IV) compound di-n-Butyl-di-(4 chlorobenzohydroxamato) tin (IV) and its probable mechanism. Molecules, 17: 10994-11009. DOI: 10.3390/molecules170910994 